Volatilomic Profiling of Citrus Juices by Dual Detection HS-GC-MS-IMS and Machine Learning - an Alternative Authentication Approach

Rebecca Brendel $^{\mathrm{a}, \mathrm{b}}$, Sebastian Schwolow ${ }^{\mathrm{a}}$, Sascha Rohn ${ }^{\mathrm{b}, \mathrm{c}}$, Philipp Weller ${ }^{\mathrm{a}, *}$

${ }^{a}$ Institute for Instrumental Analytics and Bioanalytics, Mannheim University of Applied Sciences, PaulWittsack-Straße 10, 68163 Mannheim, Germany

${ }^{b}$ Institute of Food Chemistry, Hamburg School of Food Science, University of Hamburg, Grindelallee 117, 20146 Hamburg, Germany

${ }^{\mathrm{c}}$ Department of Food Chemistry and Analysis, Institute of Food Technology and Food Chemistry, Technische Universität Berlin, TIB 4/3-1, Gustav-Meyer-Allee 25, 13355 Berlin, Germany

*Corresponding author: Tel.: +49-(0)621 292 6484, Fax: +49-(0)621 292 6420, E-mail: p.weller@hsmannheim.de. 
Table S1. Retention times, $m / z$ ratios and drift times of the reference standards.

\begin{tabular}{|c|c|c|c|c|}
\hline & $\begin{array}{c}\text { Substance } \\
\text { (molecular mass) }\end{array}$ & $\begin{array}{c}\text { Retention time } \\
\text { [min] } \\
\text { IMS/ MS }\end{array}$ & $\begin{array}{c}m / z \text { ratios (relative intensity) } \\
\text { EI-MS }\end{array}$ & $\begin{array}{l}\text { Drift times [RIPrel] } \\
\quad\left(\mathrm{K}_{0}\left[\mathrm{~cm}^{2} / \mathrm{Vs}\right]\right)^{* *}\end{array}$ \\
\hline 1 & $\begin{array}{c}\text { a-pinene } \\
(136.23 \mathrm{~g} / \mathrm{mol})\end{array}$ & $5.13 / 5.09$ & $\begin{array}{l}67(13), 77(31), 79(28), 80(11), 91(73), \\
92(46), 93(100), 105(16), 117(10), 119 \\
(18), 121(17), 132(10), 136(11)\end{array}$ & $\begin{array}{c}1.258,1.336,1.745,1.767,1.818 \\
(1.630,1.535,1.175,1.160,1.128)\end{array}$ \\
\hline 2 & $\begin{array}{c}\boldsymbol{\beta} \text {-myrcene } \\
(136.23 \mathrm{~g} / \mathrm{mol})\end{array}$ & $5.90 / 5.85$ & $\begin{array}{l}67(11), 69(47), 77(21), 79(21), 91(36), \\
92(14), 93(100), 94(11), 119(31), 121 \\
(17), 136(14)\end{array}$ & $\begin{array}{c}1.258,1.769,1.795 \\
(1.630,1.159,1.142)\end{array}$ \\
\hline 3 & $\begin{array}{c}\text { limonene } \\
(136.23 \mathrm{~g} / \mathrm{mol})\end{array}$ & $6.57 / 6.50$ & $\begin{array}{l}51(15), 53(25), 67(66), 68(100), 69(17), \\
77(22), 79(45), 91(64), 92(39), 93(70), \\
94(31), 95(14), 105(27), 107(36), 117 \\
(17), 119(30), 121(31), 132(11), 134(11), \\
136(15)\end{array}$ & $\begin{array}{c}1.258,1.336,1.736,1.795 \\
(1.630,1.535,1.181,1.142)\end{array}$ \\
\hline 4 & $\begin{array}{c}\text { linalool oxide I } \\
(170.25 \mathrm{~g} / \mathrm{mol})\end{array}$ & $7.15 / 7.05$ & $\begin{array}{l}55(44), 58(26), 59(100), 67(29), 68(38), \\
69(20), 71(17), 72(11), 77(18), 79(20), \\
81(25), 83(14), 85(10), 91(20), 93(42), \\
94(44), 97(12), 109(13), 111(38), 137 \\
(12)\end{array}$ & $\begin{array}{c}1.305 \\
(1.571)\end{array}$ \\
\hline 5 & $\begin{array}{l}\text { linalool oxide II } \\
(170.25 \mathrm{~g} / \mathrm{mol})\end{array}$ & $7.37 / 7.27$ & $\begin{array}{l}53(17), 55(38), 58(18), 59(100), 67(46), \\
68(34), 69(20), 70(12), 71(21), 77(10), \\
79(31), 81(31), 83(20), 91(24), 93(50), \\
94(53), 95(22), 97(18), 107(10), 111 \\
(28), 137(10)\end{array}$ & $\begin{array}{c}1.305 \\
(1.571)\end{array}$ \\
\hline 6 & $\begin{array}{c}\text { linalool } \\
(154.25 \mathrm{~g} / \mathrm{mol})\end{array}$ & $7.53 / 7.45$ & $\begin{array}{l}55(14), 67(16), 69(34), 71(32), 77(17), \\
79(20), 80(20), 91(33), 93(100), 94(14), \\
105(10), 107(10), 121(27), 136(17)\end{array}$ & $\begin{array}{c}1.258,1.336,1.795 \\
(1.630,1.535,1.142)\end{array}$ \\
\hline 7 & $\begin{array}{c}\text { a-terpineol } \\
(154.25 \mathrm{~g} / \mathrm{mol})\end{array}$ & $9.01 / 8.83$ & $\begin{array}{l}51(22), 53(16), 55(12), 58(23), 59(83), \\
65(17), 67(35), 68(29), 77(38), 78(10), \\
79(56), 80(14), 81(43), 91(87), 92(57), \\
93(100), 94(23), 95(20), 105(18), 107 \\
(14), 115(13), 117(15), 119(42), 121(65), \\
132(12), 134(18), 136(54)\end{array}$ & $\begin{array}{c}1.258,1.336 \\
(1.630,1.535)\end{array}$ \\
\hline 8 & carvone & $9.70 / 9.51$ & $\begin{array}{l}53(10), 54(41), 67(11), 77(22), 79(33), \\
81(10), 82(100), 91(28), 93(41), 105 \\
(37), 106(16), 107(33), 108(46), 120(15), \\
150(17)\end{array}$ & $\begin{array}{c}1.319,1.913 \\
(1.555,1.072)\end{array}$ \\
\hline 9 & $\begin{array}{l}\text { } \boldsymbol{\beta} \text {-caryophyllene* } \\
(204.36 \mathrm{~g} / \mathrm{mol})\end{array}$ & $12.03 / 11.82$ & $\begin{array}{l}55(34), 65(22), 67(40), 69(66), 77(37), \\
79(64), 81(43), 91(100), 92(31), 93(43), \\
105(65), 106(39), 107(45), 109(21), 119 \\
(34), 121(20), 131(25), 133(74), 134(24), \\
147(29), 148(26), 161(36)\end{array}$ & $\begin{array}{c}1.494,1.586 \\
(1.372,1.293)\end{array}$ \\
\hline
\end{tabular}

\footnotetext{
*The drift times are normalized to the reactant ion drift time.

**Reduced mobility $K_{0}=K\left(\frac{273}{T}\right)\left(\frac{P}{760}\right)$ with $K=\frac{L}{t_{d} E}$, where $\mathrm{L}$ is the drift tube length [cm], $\mathrm{t}_{\mathrm{d}}$ is the drift time [s], $\mathrm{E}$ is the electrical field $[\mathrm{V} / \mathrm{cm}], \mathrm{T}$ is the drift tube temperature $[\mathrm{K}]$ and $\mathrm{P}$ is the pressure inside the drift tube [Torr]
} 

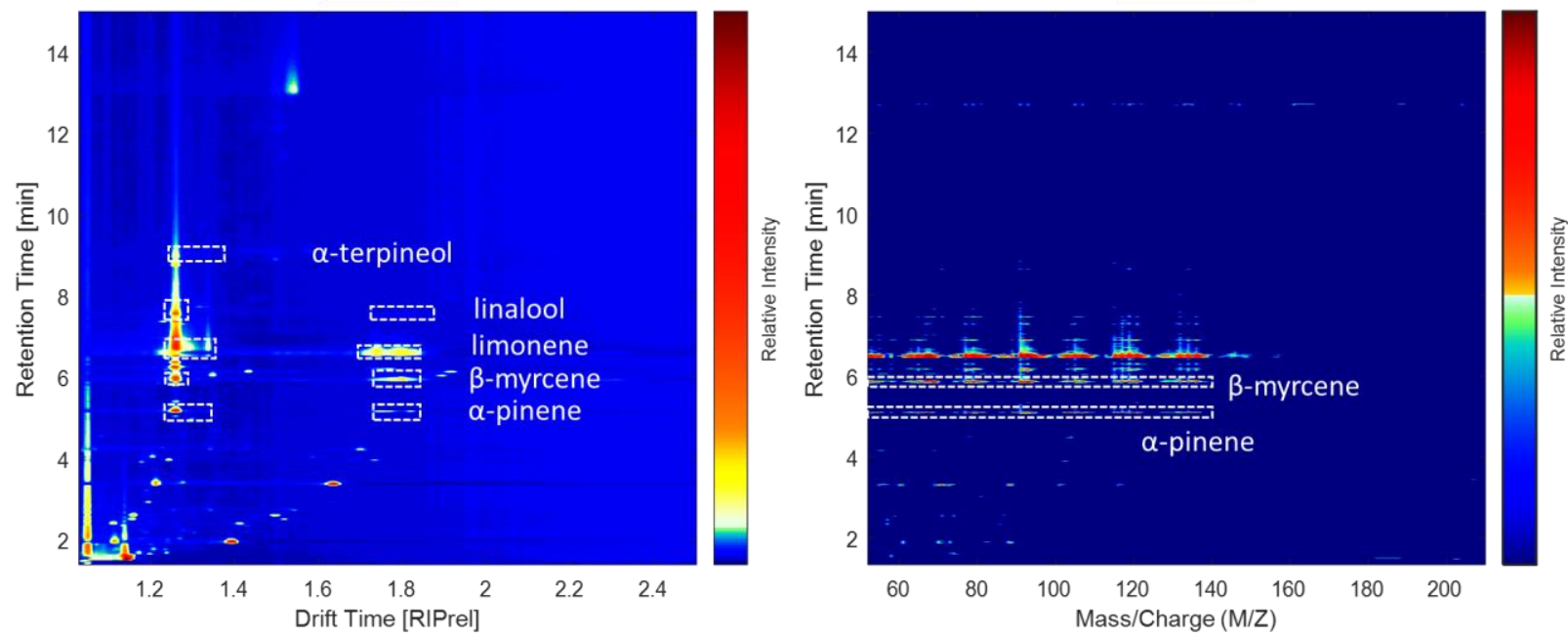

Figure S1. IMS (left) and MS (right) spectrum of Sample 22 (blood orange juice not from fruit juice concentrate (NFC)) with identified substances marked in white, dashed boxes.
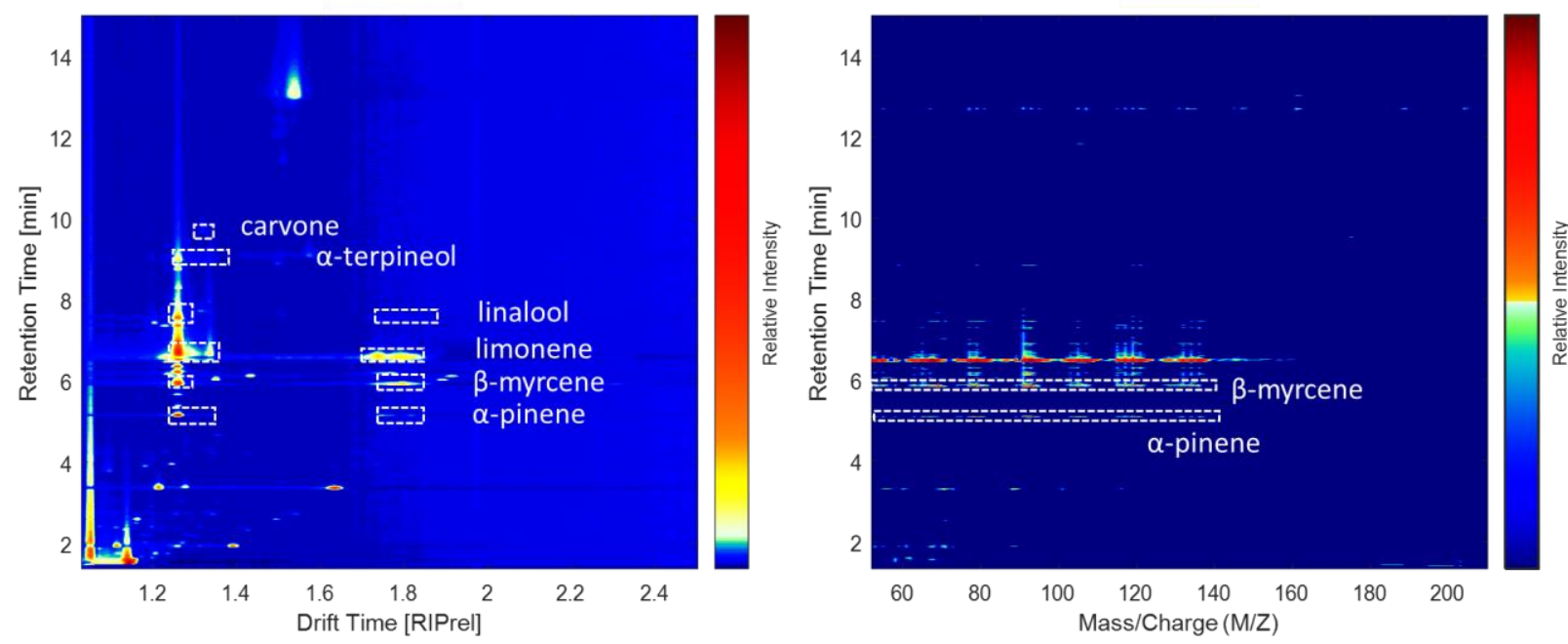

Figure S2. IMS (left) and MS (right) spectrum of Sample 1 (orange juice not from fruit juice concentrate (NFC)) with identified substances marked in white, dashed boxes. 

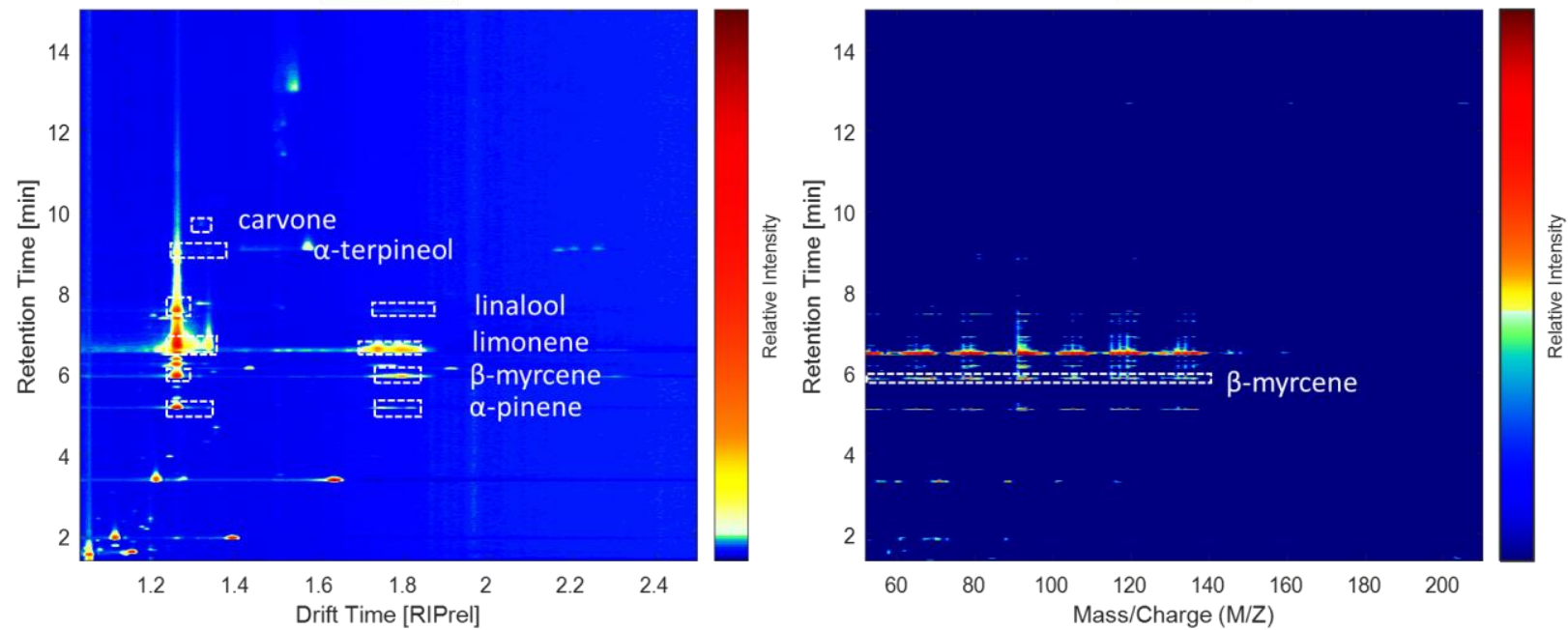

Figure S3. IMS (left) and MS (right) spectrum of Sample 23 (orange juice from fruit juice concentrate (FC)) with identified substances marked in white, dashed boxes.
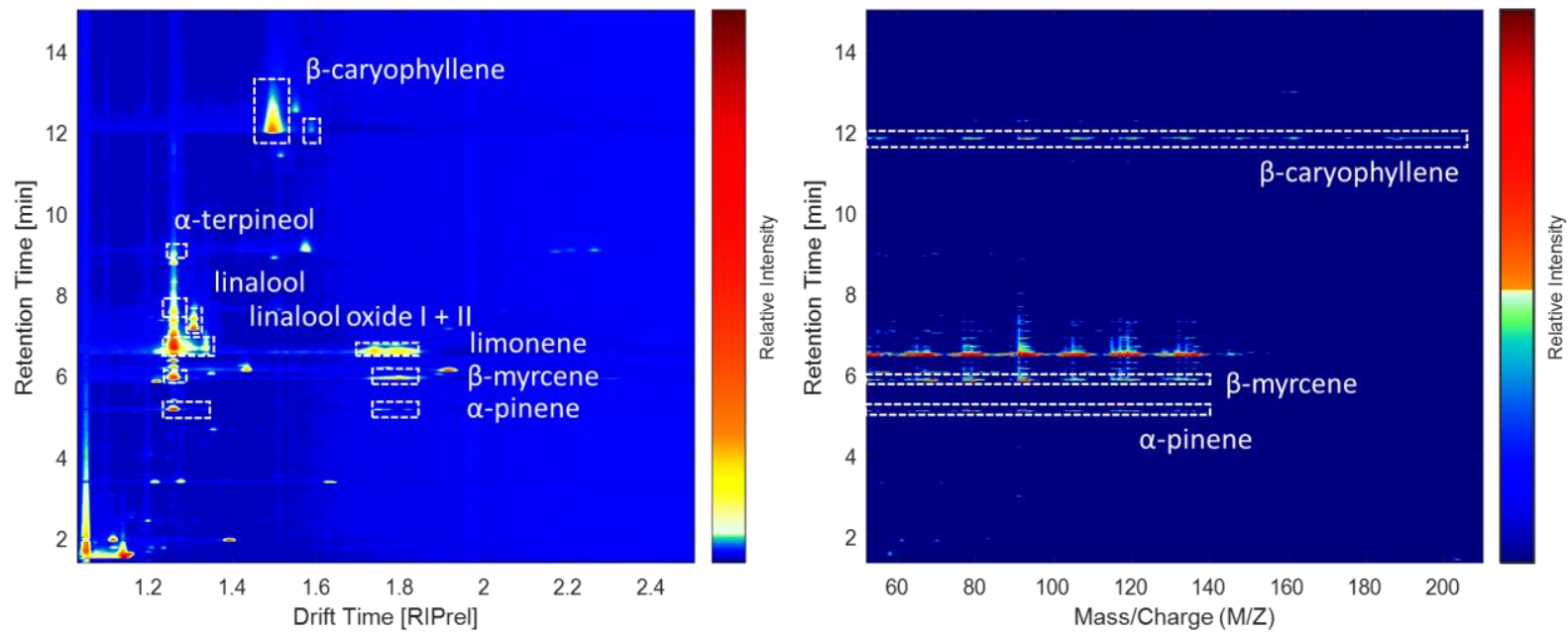

Figure S4. IMS (left) and MS (right) spectrum of Sample 6 (grapefruit juice not from fruit juice concentrate (NFC)) with identified substances marked in white, dashed boxes. 

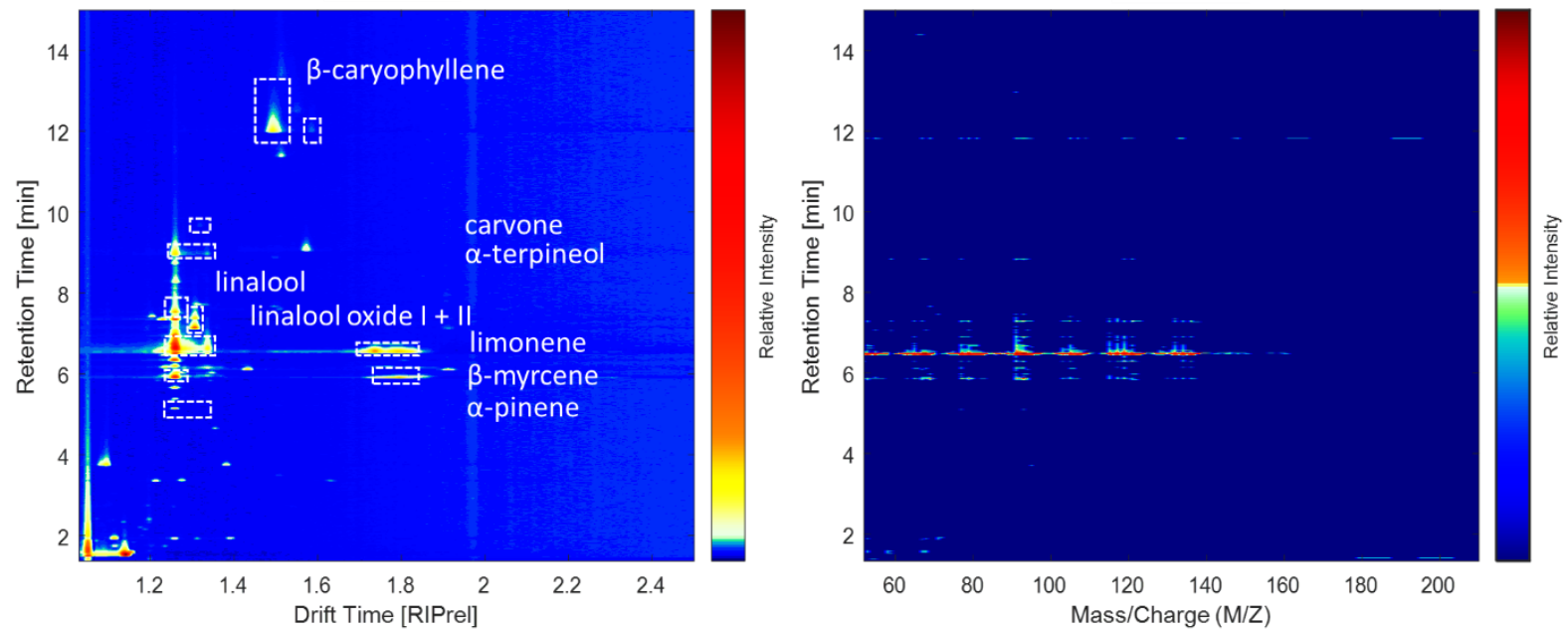

Figure S5. IMS (left) and MS (right) spectrum of Sample 2 (grapefruit juice from fruit juice concentrate (FC)) with identified substances marked in white, dashed boxes.

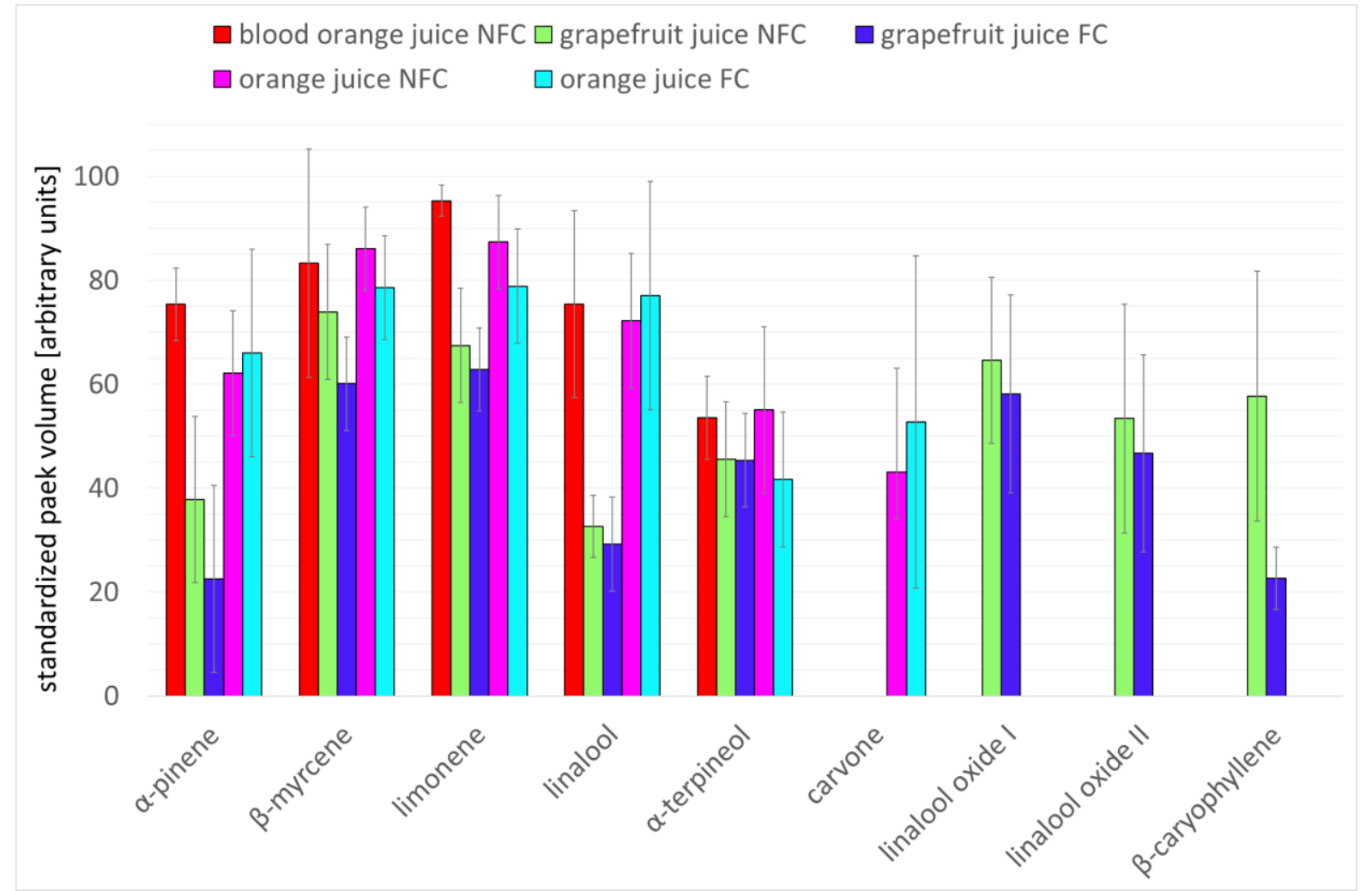

Figure S6. Arithmetic mean with standard deviation error bars of the standardized peak volumes of the identified substances calculated for each juice class, respectively. For standardization, the peak volume of each substance was divided by the highest peak volume obtained for the respective substance in all samples and multiplied by a factor of 100 (NFC = not from fruit juice concentrate; $F C=$ from fruit juice concentrate). 
Table S2. Standardized peak volumes of the identified substances in all investigated samples.

\begin{tabular}{|c|c|c|c|c|c|c|c|c|c|c|}
\hline \multirow[b]{2}{*}{$\begin{array}{c}\text { Sample } \\
\text { name }\end{array}$} & \multirow[b]{2}{*}{ Juice class } & \multicolumn{9}{|c|}{ Substance } \\
\hline & & $\mathbf{I}^{*}$ & II* & III* & IV* & $\mathbf{V}^{*}$ & VI* & VII* & VIII* & IX* \\
\hline 1 & $\begin{array}{l}\text { orange juice } \\
\text { NFC }\end{array}$ & 54 & 88 & 80 & 70 & 55 & 52 & n.d. $* *$ & n.d. & n.d. \\
\hline 2 & grapefruit FC & 7 & 71 & 61 & 33 & 60 & 40 & 56 & 46 & 30 \\
\hline 3 & grapefruit NFC & 26 & 70 & 56 & 27 & 50 & n.d. & 58 & 50 & 32 \\
\hline 5 & $\begin{array}{c}\text { orange juice } \\
\text { NFC }\end{array}$ & 76 & 94 & 96 & 83 & 45 & 55 & n.d. & n.d. & n.d. \\
\hline 6 & grapefruit NFC & 57 & 83 & 78 & 36 & 33 & n.d. & 78 & 71 & 92 \\
\hline 7 & grapefruit NFC & 35 & 81 & 69 & 35 & 63 & n.d. & 63 & 55 & 44 \\
\hline 8 & orange juice $\mathrm{FC}$ & 30 & 69 & 58 & 32 & 33 & n.d. & n.d. & n.d. & n.d. \\
\hline 9 & orange juice FC & 37 & 71 & 59 & 49 & 25 & n.d. & n.d. & n.d. & n.d. \\
\hline 10 & $\begin{array}{c}\text { orange juice } \\
\text { NFC }\end{array}$ & 56 & 100 & 93 & 58 & 100 & n.d. & n.d. & n.d. & n.d. \\
\hline 11 & $\begin{array}{c}\text { orange juice } \\
\text { NFC }\end{array}$ & 74 & 89 & 96 & 83 & 43 & 51 & n.d. & n.d. & n.d. \\
\hline 12 & $\begin{array}{c}\text { orange juice } \\
\text { NFC }\end{array}$ & 52 & 79 & 76 & 68 & 39 & 48 & n.d. & n.d. & n.d. \\
\hline 13 & $\begin{array}{c}\text { orange juice } \\
\text { NFC }\end{array}$ & 51 & 81 & 77 & 67 & 43 & 42 & n.d. & n.d. & n.d. \\
\hline 14 & $\begin{array}{l}\text { orange juice } \\
\text { NFC }\end{array}$ & 60 & 85 & 82 & 81 & 63 & 44 & n.d. & n.d. & n.d. \\
\hline 15 & grapefruit NFC & 18 & 68 & 57 & 30 & 52 & n.d. & 60 & 41 & 36 \\
\hline 16 & $\begin{array}{l}\text { orange juice } \\
\text { NFC }\end{array}$ & 75 & 93 & 99 & 80 & 63 & 48 & n.d. & n.d. & n.d. \\
\hline 17 & $\begin{array}{l}\text { orange juice } \\
\text { NFC }\end{array}$ & 39 & 81 & 75 & 38 & 63 & n.d. & n.d. & n.d. & n.d. \\
\hline 18 & $\begin{array}{l}\text { blood orange } \\
\text { NFC }\end{array}$ & 76 & 93 & 98 & 90 & 63 & n.d. & n.d. & n.d. & n.d. \\
\hline 19 & $\begin{array}{l}\text { blood orange } \\
\text { NFC }\end{array}$ & 79 & 94 & 100 & 87 & 56 & 43 & n.d. & n.d. & n.d. \\
\hline 20 & $\begin{array}{c}\text { orange juice } \\
\text { NFC }\end{array}$ & 63 & 86 & 86 & 80 & 44 & 51 & n.d. & n.d. & n.d. \\
\hline 21 & $\begin{array}{l}\text { blood orange } \\
\text { NFC }\end{array}$ & 76 & 93 & 96 & 85 & 60 & n.d. & n.d. & n.d. & n.d. \\
\hline 22 & $\begin{array}{c}\text { blood orange } \\
\text { NFC }\end{array}$ & 82 & 94 & 99 & 87 & 57 & n.d. & n.d. & n.d. & n.d. \\
\hline 23 & orange juice FC & 70 & 83 & 83 & 79 & 34 & 41 & n.d. & n.d. & n.d. \\
\hline 24 & $\begin{array}{c}\text { orange juice } \\
\text { NFC }\end{array}$ & 72 & 92 & 97 & 81 & 60 & 65 & n.d. & n.d. & n.d. \\
\hline 29 & orange juice FC & 72 & 75 & 77 & 87 & 42 & 72 & n.d. & n.d. & n.d. \\
\hline 30 & orange juice FC & 70 & 74 & 86 & 83 & 42 & 73 & n.d. & n.d. & n.d. \\
\hline 31 & orange juice FC & 69 & 76 & 86 & 90 & 67 & 73 & n.d. & n.d. & n.d. \\
\hline 32 & $\begin{array}{l}\text { orange juice } \\
\text { NFC }\end{array}$ & 79 & 80 & 99 & 84 & 53 & 45 & n.d. & n.d. & n.d. \\
\hline 33 & $\begin{array}{c}\text { blood orange } \\
\text { NFC }\end{array}$ & 77 & 80 & 92 & 62 & 60 & n.d. & n.d. & n.d. & n.d. \\
\hline
\end{tabular}




\begin{tabular}{|c|c|c|c|c|c|c|c|c|c|c|}
\hline \multirow[b]{2}{*}{$\begin{array}{c}\text { Sample } \\
\text { name }\end{array}$} & \multirow[b]{2}{*}{ Juice class } & \multicolumn{9}{|c|}{ Substance } \\
\hline & & $I^{*}$ & II* & III* & IV* & $\mathbf{V}^{*}$ & VI* & VII* & VIII* & $\mathbf{I X}^{*}$ \\
\hline 34 & $\begin{array}{c}\text { orange juice } \\
\text { NFC }\end{array}$ & 57 & 71 & 79 & 64 & 45 & 60 & n.d. & n.d. & n.d. \\
\hline 35 & $\begin{array}{c}\text { blood orange } \\
\text { NFC }\end{array}$ & 75 & 92 & 95 & 97 & 50 & 37 & n.d. & n.d. & n.d. \\
\hline 36 & grapefruit FC & n.d. & n.d. & n.d. & 15 & n.d. & n.d. & 34 & 22 & n.d. \\
\hline 37 & orange juice FC & 70 & 71 & 83 & 83 & 33 & 65 & n.d. & n.d. & n.d. \\
\hline 38 & orange juice FC & 66 & 74 & 85 & 98 & 40 & 48 & n.d. & n.d. & n.d. \\
\hline 39 & $\begin{array}{c}\text { blood orange } \\
\text { NFC }\end{array}$ & 81 & 99 & 94 & 65 & 54 & n.d. & n.d. & n.d. & n.d. \\
\hline 40 & grapefruit NFC & 65 & 88 & 86 & 42 & 34 & n.d. & 60 & 50 & 100 \\
\hline 41 & grapefruit NFC & 30 & 70 & 59 & 31 & 40 & n.d. & 65 & 54 & 49 \\
\hline 42 & orange juice FC & 100 & 97 & 86 & 69 & 38 & 55 & n.d. & n.d. & n.d. \\
\hline 43 & orange juice FC & 76 & 95 & 86 & 100 & 62 & 100 & n.d. & n.d. & n.d. \\
\hline 44 & grapefruit FC & 16 & 60 & 61 & 33 & 43 & n.d. & 71 & 57 & 29 \\
\hline 45 & grapefruit FC & 53 & 86 & 76 & 41 & 48 & n.d. & 88 & 78 & 20 \\
\hline 46 & grapefruit FC & 32 & 72 & 60 & 27 & 38 & n.d. & 53 & 41 & 19 \\
\hline 47 & $\begin{array}{l}\text { blood orange } \\
\text { NFC }\end{array}$ & 59 & 28 & 90 & 43 & 39 & n.d. & n.d. & n.d. & n.d. \\
\hline 48 & $\begin{array}{l}\text { blood orange } \\
\text { NFC }\end{array}$ & 74 & 77 & 95 & 62 & 44 & n.d. & n.d. & n.d. & n.d. \\
\hline 49 & grapefruit NFC & 31 & 56 & 60 & 27 & 44 & n.d. & 53 & 33 & 46 \\
\hline 50 & grapefruit NFC & 25 & 58 & 65 & 25 & 36 & n.d. & 44 & 28 & 54 \\
\hline 51 & grapefruit FC & 26 & 71 & 56 & 27 & 37 & n.d. & 47 & 36 & 15 \\
\hline 52 & grapefruit NFC & 53 & 91 & 80 & 41 & 59 & n.d. & 100 & 100 & 65 \\
\hline
\end{tabular}

*I = $\alpha$-terpineol; II = $\beta$-myrcene; III = limonene; IV = linalool; V= $\alpha$-terpineole, $\mathrm{VI}=$ carvone; $\mathrm{VII}=$ linalool oxide I; VIII = linalool oxide II; IX = $\beta$-caryophyllene.

$* *$ n.d. $=$ not detected. 


\section{Figures of merit:}

- $\quad$ classification ability $(\mathrm{CA})=\frac{\sum c_{\text {train }}}{n} * 100$,

where $c_{\text {train }}$ is the sum of correct classified samples of the training set, and $n$ the number of all training set samples.

- $\quad$ prediction ability $(\mathrm{PA})=\frac{\sum c_{t e s t}}{n} * 100$

where $c_{\text {test }}$ is the sum of correct classified samples of the test set, and $n$ the number of all test set samples.

- Prediction ability for class $x=\frac{\sum c_{t e s t, x}}{n_{x}} * 100$

where $c_{t e s t, x}$ is the sum of correct classified samples of the test set belonging to class $x$, and $n_{x}$ the number of all test set samples belonging to class $x$. 


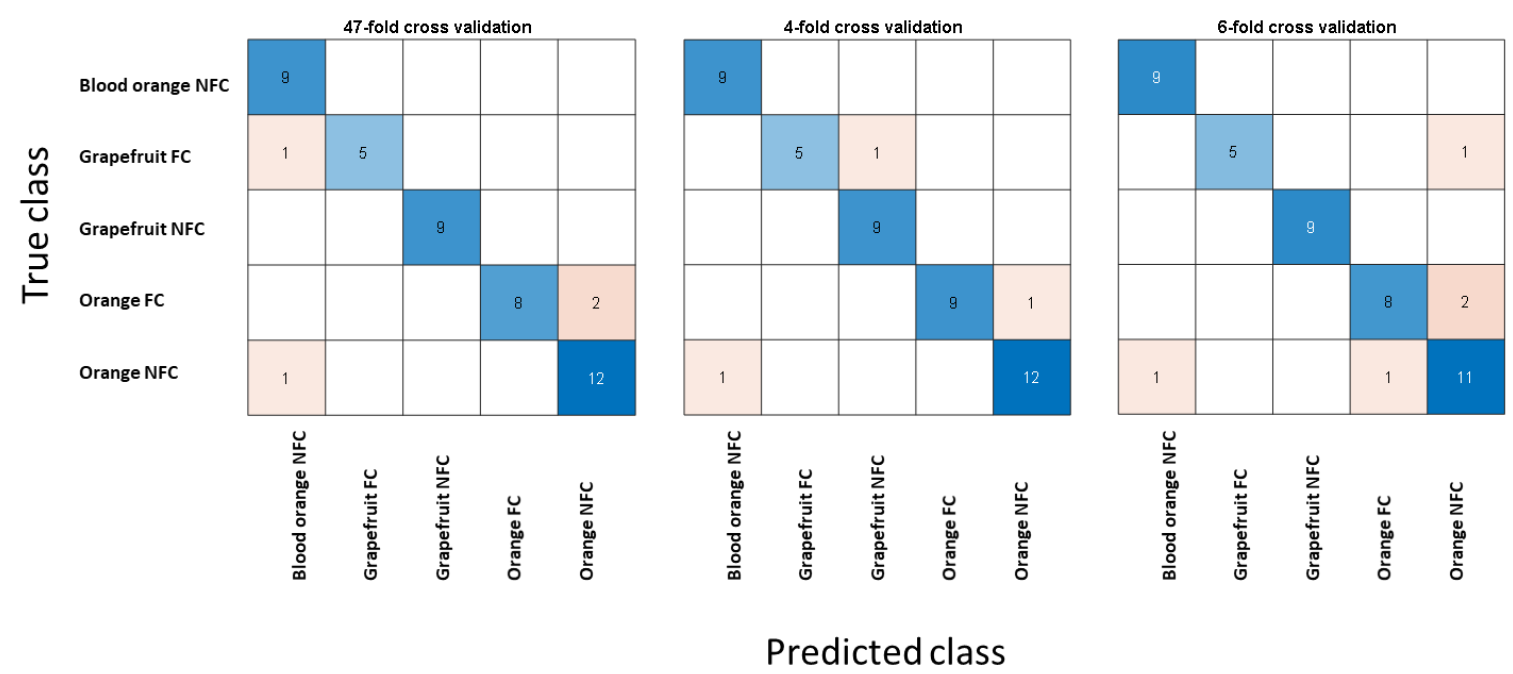

Figure S7. Confusion matrices obtained by leave-one out, 4-fold and 6-fold cross-validation (left to right) for PCALDA applied to the IMS data.

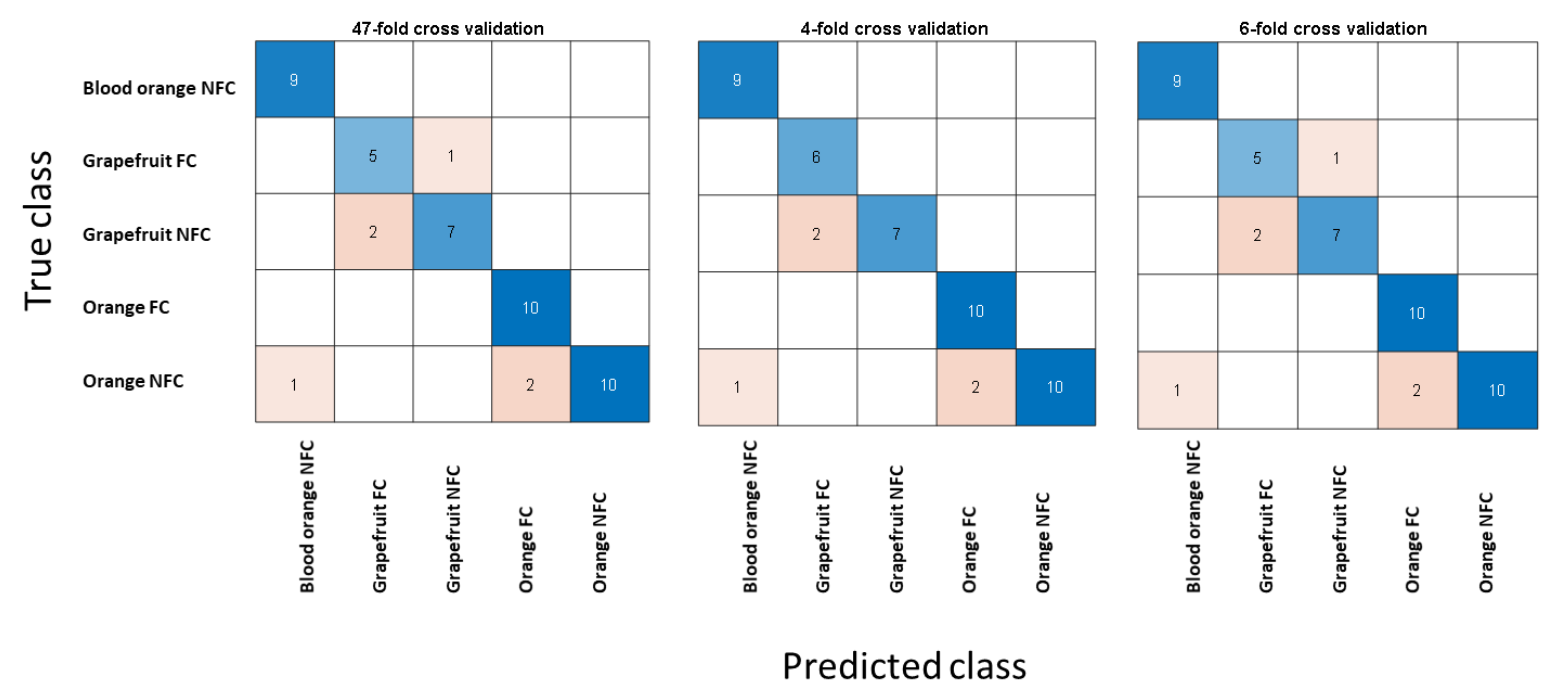

Figure S8. Confusion matrices obtained by leave-one out, 4-fold and 6-fold cross-validation (left to right) for PCALDA applied to the MS data. 


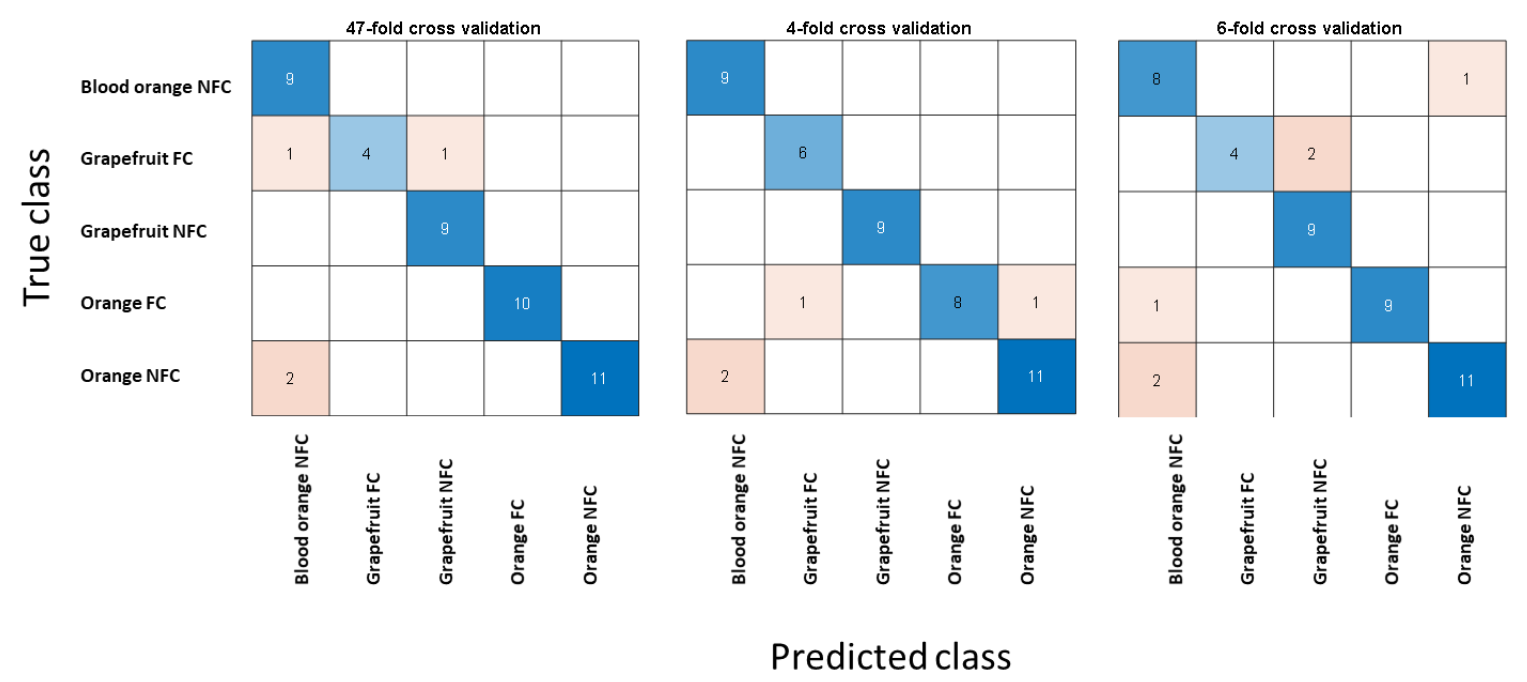

Figure S9. Confusion matrices obtained by leave-one out, 4-fold and 6-fold cross-validation (left to right) for PCALDA applied after low-level data fusion.

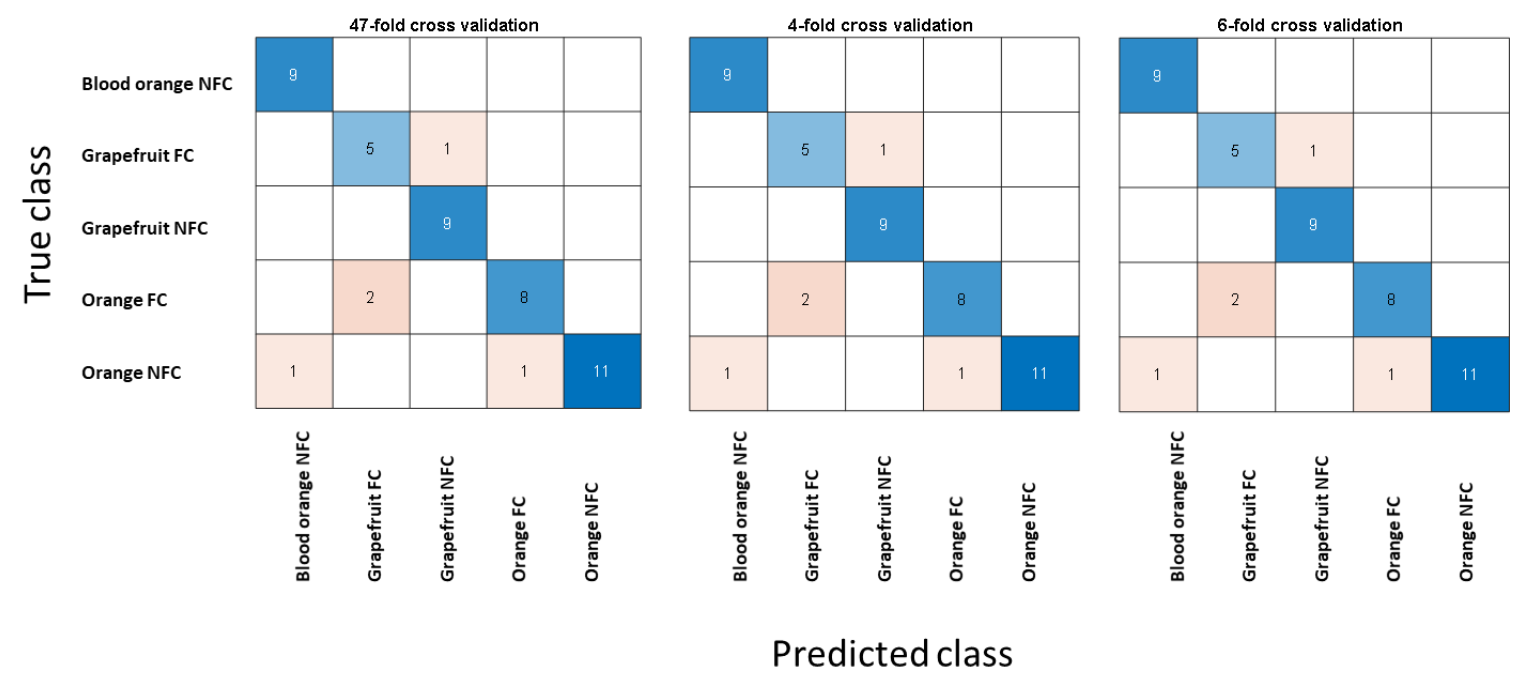

Figure 10. Confusion matrices obtained by leave-one out, 4-fold and 6-fold cross-validation (left to right) for PCA-LDA applied after mid-level data fusion. 